EPJ Web of Conferences 53, 05002 (2013)

DOI: $10.1051 /$ epjconf/20135305002

(C) Owned by the authors, published by EDP Sciences, 2013

\title{
Search for ultra-high energy photons at the Pierre Auger Observatory
}

\author{
V. Scherini ${ }^{1, a}$ for the Pierre Auger Collaboration ${ }^{2, b}$ \\ 1 Università degli Studi di Milano and INFN Milano, via Celoria 16, 20133 Milano, Italy \\ 2 Observatorio Pierre Auger, Av. San Martín Norte 304, 5613 Malargüe, Argentina \\ (Full author list: http://www.auger.org/archive/authors_2012_06.html)
}

\begin{abstract}
The Pierre Auger Observatory has a significant sensitivity to search for ultra-high energy photons by using data from the ground detector alone or in combination with the fluorescence detector (hybrid mode). Recent upper limits on the photon flux are below the percent-level with regard to the total flux of cosmic rays. The current status and the details of the analysis methods are presented.
\end{abstract}

\section{INTRODUCTION}

All scenarios invoked to explain the origin of Ultra-High Energy Cosmic Rays (UHECR) predict fractions of photons along with the nuclear component. The expected fluxes at the Earth depend primarily on the chemical composition of UHECRs and on the nature, the cosmological evolution and the spatial distribution of astrophysical sources [1,2].

A guaranteed flux of photons is expected from the interaction of high energy particles with background radiation fields. In particular, cosmic rays above $\sim 4 \cdot 10^{19} \mathrm{eV}\left(10^{18} \mathrm{eV}=1 \mathrm{EeV}\right)$ propagating through the cosmic microwave background may exceed the threshold for resonant $\Delta+$ particle production, the so-called Greisen-Zatsepin-Kuz'min (GZK) process [3]. A large fraction $(\sim 50 \%)$ of photons in the cosmic-ray spectrum at the highest energies was predicted within the socalled "top-down" (i.e. non-acceleration) models [4] to explain the origin of trans-GZK cosmic rays. Severe constraints to these models were imposed by limits derived in previous photon searches [5-11].

UHE photon detection at Earth would open a new window to the Universe. Photons point back to the sites of production, revealing details of the sources and of their acceleration mechanisms and providing a different view on UHECRs origin, complementary with respect to charged particles. They would also deliver information on extragalactic radio background and magnetic fields. Finally, by probing aspects of high-energy interactions, their detection would have large impact on fundamental physics [12]. A comprehensive review on the status of the art of photon searches can be found in [13].

\section{PHOTON SEARCH AT THE PIERRE AUGER OBSERVATORY}

The Pierre Auger Observatory [14], located near Malargüe, Argentina, consists of a Surface Detector Array (SD) [15] of 1660 water Cherenkov stations spread over an area of $3000 \mathrm{~km}^{2}$ and overlooked by the 27 telescopes of the Fluorescence Detector (FD) [16]. The SD samples the density of the secondary particles of the shower at the ground while the FD observes the shower longitudinal development in

\footnotetext{
ae-mail: viviana.scherini@mi.infn.it

${ }^{b}$ For the full authorlist see Appendix "Collaborations" in this volume

This is an Open Access article distributed under the terms of the Creative Commons Attribution License 2.0, which permits unrestricted use, distribution, and reproduction in any medium, provided the original work is properly cited.
} 


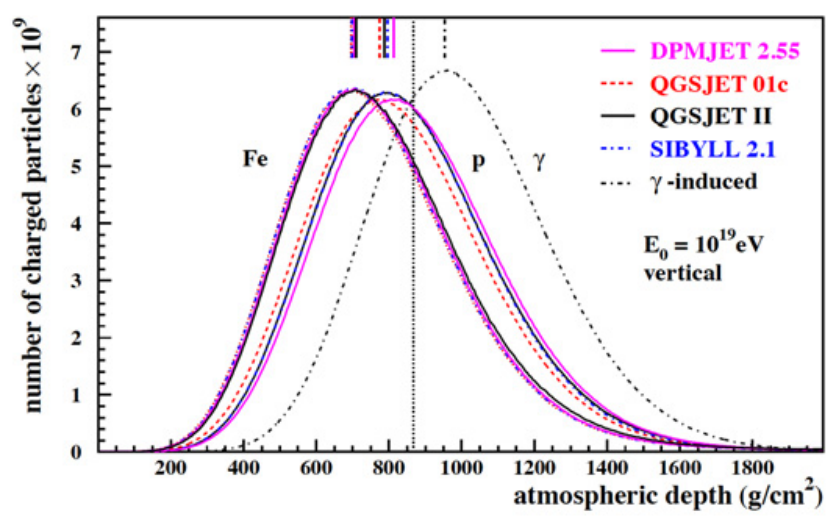

Figure 1. Average longitudinal profile for Monte Carlo showers induced by photon and nuclear primaries for different hadronic interaction models. The average depth of the shower maximum, $X_{\max }$, differs by more than $\sim 200 \mathrm{~g} \mathrm{~cm}^{-2}$ at energies around $10 \mathrm{EeV}$.

the atmosphere. The large collection area and the availability of a variety of composition-sensitive parameters provide an excellent opportunity to search for photons in the cosmic ray flux above $1 \mathrm{EeV}$.

\subsection{Fluorescence Detector observables}

The key observable in searches for photon primaries with the Auger fluorescence detector is the depth of shower maximum, $X_{\max }$. Since the fluorescence telescopes follow the longitudinal shower development, the depth of maximum can be measured with very high accuracy.

Deeper shower maximum values are expected for photon showers due to the smaller multiplicity in electromagnetic interactions, with respect to hadronic ones. The LPM effect [17] can even further delay the shower development above $10 \mathrm{EeV}$. In Fig. 1 the average longitudinal profile is plotted for samples of Monte Carlo showers induced by photons and nuclear primaries (protons and iron nuclei for different hadronic interaction models) at $10 \mathrm{EeV}$. The difference between the average $X_{\max }$ value for showers induced by protons and photons at this energy is $\sim 200 \mathrm{~g} \mathrm{~cm}^{-2}$, which is large compared to $X_{\max }$ resolution of the hybrid detector, $\sim 20 \mathrm{~g} \mathrm{~cm}^{-2}$ [18].

Upper limits on the photon fraction in $\mathrm{CR}$ above $10 \mathrm{EeV}$ were derived by comparing the measured depth of shower maximum to the Monte Carlo expectations for showers of the same geometry and energy, but assuming a primary photon origin [9]. Due to fluctuations of the nuclear background, with an increased statistics photon candidates can appear in the bulk of hadron-like data. By comparing their number with the total number of events, and taking into account the detection efficiencies for different primaries, upper limit were extended in the EeV range [11], see Fig. 4 (label Hyb 2009).

\subsection{Surface detector observables}

Observables of the surface detector array, directly connected to the cascade development are: the risetime, $\mathrm{t}_{1 / 2}$, of the signal at $1000 \mathrm{~m}$ from shower core, and the curvature of the shower front. Due to the geometry associated with particles in the shower, the distribution of arrival times of particles is expected to become more dispersed with increasing $X_{\max }$. Moreover the arrival time of the first particles at a given distance from the shower axis is expected to be delayed with respect to a planar shower front. With increasing $X_{\max }$, this delay increases, and the radius of curvature is thus expected to decrease for photon induced showers. 

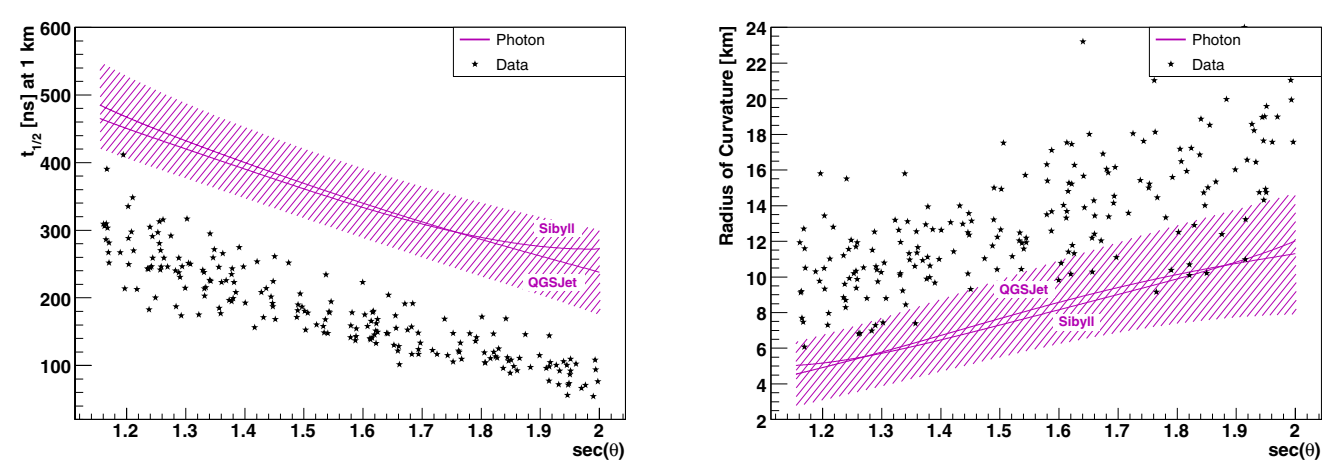

Figure 2. Discriminating observables for the Auger surface detector [10]. Left panel: signal rise-time $\mathrm{t}_{1 / 2}$; right panel: radius of curvature of the shower front. The parametrization of the mean behavior for $20 \mathrm{EeV}$ primary photons is plotted as a function of shower zenith angle along with a sample of data.

A detailed Monte Carlo study was performed to characterize the behavior of photon induced showers. In Fig. 2 the shower front curvature and the signal rise-time are shown for photon simulations and data. A combination of the two observables can be obtained via principal component analysis on photon simulations and on a sub-sample $(\sim 5 \%)$ of recorded data. Photon candidates were selected in the remaining data with a cut determined "a priori" at the median of the obtained distributions for photon induced showers. Upper limits on the absolute photon flux were derived by relating the number of photon-like events to the well known experimental exposure [10], see Fig. 4 (label SD).

Below $10 \mathrm{EeV}$, the requirement of a large footprint and large signals in the individual stations, needed for a reliable calculation of rise-time and radius of curvature, is not met by photon showers. A further observable, the $S_{b}$ parameter [19], has been introduced combining the different amplitude of the signal in the surface detector and the steeper lateral distribution function (i.e. the signals recorded in the SD stations as a function of distance from the axis) expected for photon induced showers. It can be defined as:

$$
S_{b}=\sum_{i} S_{i}\left(\frac{R_{i}}{R_{\text {ref }}}\right)^{b}
$$

where the sum runs over the triggered stations, $S_{i}$ is the recorded signal in the i-th station at distance $R_{i}$ from the reconstructed axis and $R_{\mathrm{ref}}$ is a reference distance equal to $1000 \mathrm{~m}$ for this analysis. The exponent $b$ is chosen equal to 4 for maximizing the separation power between photons and hadrons.

\section{THE HYBRID APPROACH: COMBINATION OF OBSERVABLES}

A new approach has been developed to fully exploit the capabilities of the Pierre Auger Observatory hybrid detector in the EeV energy range. The analysis, presented in [20], is based on three elements: the accurate selection of hybrid data, a powerful statistical method combining FD and SD observables, the realistic detector exposure calculation.

\subsection{Data selection}

Hybrid events, collected between January 2005 and September 2010, with zenith angle smaller than $60^{\circ}$ and with a good geometry reconstruction are selected for the analysis. To ensure a reliable profile reconstruction the following selection criteria are applied: a reduced $\chi^{2}$ of the longitudinal profile fit to the Gaisser-Hillas function smaller than 2.5 , a $\chi^{2}$ of a linear fit to the longitudinal profile exceeding the Gaisser-Hillas fit $\chi^{2}$ by at least a factor of 1.1 , the $X_{\max }$ observed within the field of view of the 

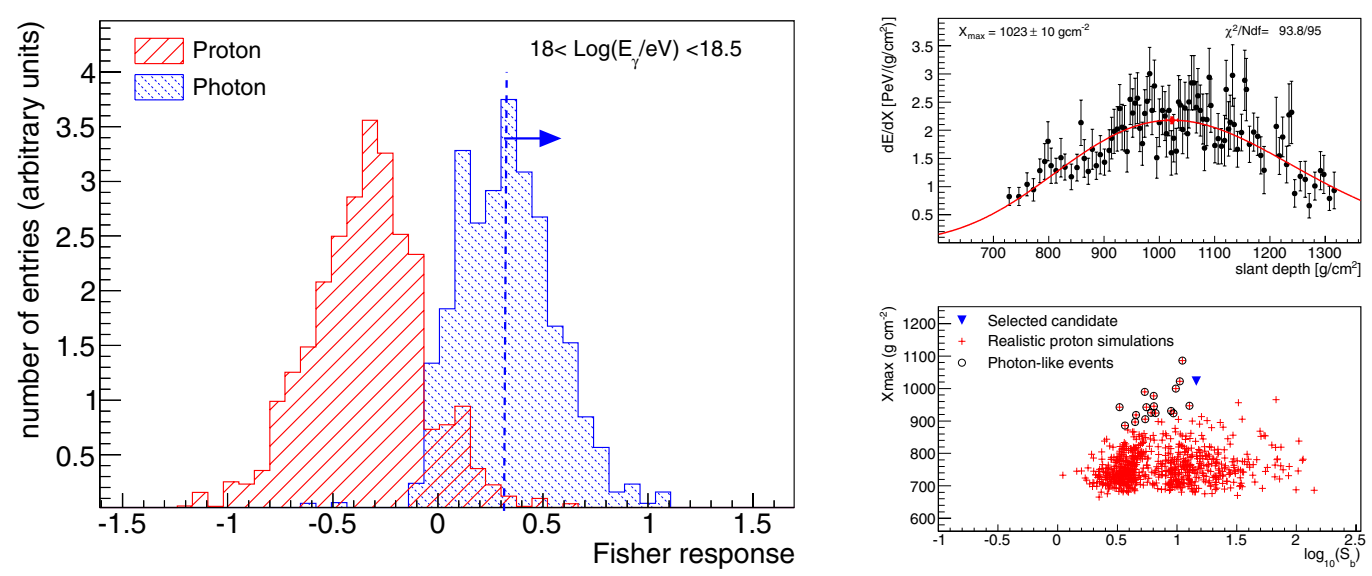

Figure 3. Left panel: distribution of the Fisher response for Monte Carlo protons (red) and photons (blue) for $18<\log (\mathrm{E} / \mathrm{eV})<18.5$ [20]. Photon-like events have a Fisher value larger than the median value of the distribution for photons, $\mathrm{X}_{c u t}$ (dashed line). Right panel: longitudinal profile and Gaisser-Hillas fit for a photon candidate (top); $X_{\max }$ vs. $\log _{10}\left(S_{b}\right)$ (bottom) for the measured event (triangle) compared to dedicated proton simulations (crosses). For this particular candidate, about $2 \%$ of such events passes the cut on the Fisher observable (empty circles) [20].

telescopes, the Cherenkov light contamination smaller than $50 \%$ and the uncertainty on the reconstructed energy within $20 \%$. Finally time periods with the sky not obscured by clouds and with a reliable measurement of the vertical optical depth of aerosols [21, 22], are selected. On the SD side the only requirements is that at least 4 stations within $2 \mathrm{~km}$ from the hybrid reconstructed axis should be active. This prevents an underestimation of $S_{b}$ (which would mimic the behavior of a photon event) due to not deployed or temporarily inefficient detectors.

\subsection{Monte Carlo studies}

To improve the photon-hadron discrimination power, the previous hybrid analyses, based on the FD $X_{\max }$ measurement, are complemented with the SD observable $S_{b}$. For the classification of photon candidates a Fisher analysis is performed. The training sample contains a total of $\sim 30000$ photon and proton CORSIKA [23] showers generated according to a power law spectrum (index =-1) between $10^{17}$ and $10^{20} \mathrm{eV}$ with QGSJET-II [24] and FLUKA [25] as high- and low-energy hadronic interaction models. To carefully reproduce the operating conditions of the data acquisision, time dependent simulations are performed according to the hybrid detector on-time. The actual configurations of FD and SD and the realistic atmospheric conditions are also taken into account. An additional independent sample of 20000 photon showers is used for determining the exposure of the hybrid detector using a procedure as the one discussed in [26].

The Fisher response is shown in Fig. 3, left panel. Photon-like events are selected by applying an "a priori" cut at $50 \%$ of the photon selection efficiency. With this choice the expected nuclear contamination is about $1 \%$ in the lowest energy interval (between $10^{18}$ and $10^{18.5} \mathrm{eV}$ ) and it becomes smaller for increasing energies.

\subsection{Upper limits on photon flux}

Applying the method to data, 6, 0, 0, 0 and 0 photon candidates are found for energies above 1, 2, 3, 5 and $10 \mathrm{EeV}$. The observed number of photon candidates is consistent with the expectation from nuclear primaries, within the assumption of a mixed composition. For the two events with the deepest $X_{\max }$ 
UHECR 2012

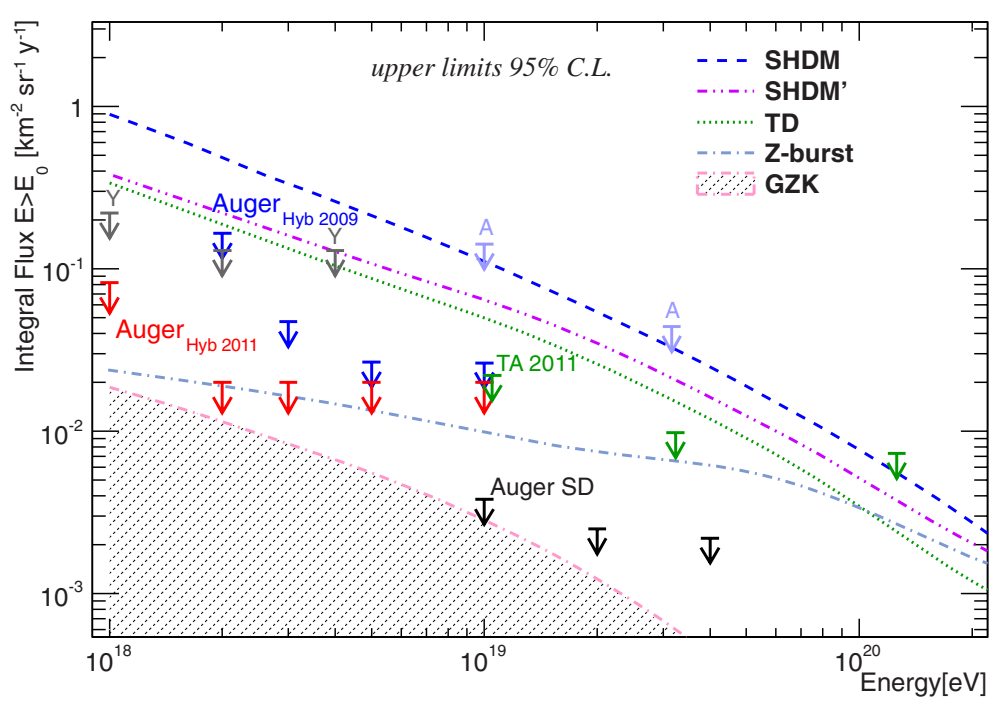

Figure 4. Upper limits on the integral photon flux by the Pierre Auger Observatory (Auger ${ }_{H y b 2011}$ [20], (Auger $_{H y b 2009}$ [11] and (Auger ${ }_{S D}$ [10]), along with the ones by other experiments (AGASA [5], Yakutsk [7] and TA [30]). Also shown are the GZK [1] and "top-down" predictions: super-heavy dark matter (SHDM [1] and SHDM' [31]), topological defects (TD) [1] and Z-bursts [1].

(both larger than $1000 \mathrm{~g} \mathrm{~cm}^{-2}$ ) the nuclear background has been individually checked by simulating 1000 dedicated proton CORSIKA showers with the same energy, arrival direction and core position as reconstructed for the real events. The profile of one candidate is shown in Fig. 3 (right panel, top).

The values of $X_{\max }$ and $S_{b}$ are compared to the expectation for protons (right panel, bottom). For this particular candidate, a fraction of about $2 \%$ of such background events passes the cut on the Fisher observable (empty circles).

The $95 \%$ CL upper limits on the photon flux $\Phi_{\gamma}^{95 C L}$ integrated above an energy threshold $E_{0}$ is given by:

$$
\Phi_{\gamma}^{95 C L}=\frac{N_{\gamma}^{95 C L}\left(E_{\gamma}>E_{0}\right)}{\mathcal{E}_{\gamma, \text { min }}} .
$$

where $E_{\gamma}$ is the reconstructed photon energy, $N_{\gamma}^{95 C L}$ is the number of photon candidates above $E_{0}$ at $95 \%$ of confidence level and $\mathcal{E}_{\gamma, \text { min }}$ is the exposure of the hybrid detector. To be conservative, in equation (2) we use the minimum value of the exposure above $E_{0}$ and a possible nuclear background is not subtracted for the calculation of $N_{\gamma}^{95 C L}$.

Upper limits on the integral photon flux of $8.2 \cdot 10^{-2} \mathrm{~km}^{-2} \mathrm{sr}^{-1} \mathrm{y}^{-1}$ above $1 \mathrm{EeV}$ and of 2.0 . $10^{-2} \mathrm{~km}^{-2} \mathrm{sr}^{-1} \mathrm{y}^{-1}$ above 2, 3, 5 and $10 \mathrm{EeV}$ are derived [20]. They are shown in Fig. 4 (label Hyb 2011) along with previous experimental results. Comparing the flux limits to the measured Auger spectrum [27], upper bounds on the fraction of photons of about $0.4 \%, 0.5 \%, 1.0 \%, 2.6 \%$ and $8.9 \%$ are obtained for energies above 1,2,3,5 and $10 \mathrm{EeV}$.

The robustness of the results was studied against different sources of uncertainty. Increasing (reducing) all $X_{\max }$ values by the uncertainty $\Delta X_{\max }=13 \mathrm{~g} \mathrm{~cm}^{-2}$ [28] changes the number of photon candidates above $1 \mathrm{EeV}$ by $+1(-2)$ not affecting the higher energies. As a consequence the upper limit above $1 \mathrm{EeV}$ increases of $\sim 10 \%$ (decreases of $\sim 25 \%$ ). The uncertainty on the shower geometry determination corresponds to $\Delta S_{b} \sim 5 \%$, changing the number of photon candidates by $\pm 0(+1)$ above $1 \mathrm{EeV}$. The overall uncertainty on the hybrid exposure calculation for photons is about $5 \%$. Another contribution comes from the uncertainty on the energy scale which has been estimated to be about 
$22 \%$ [29]. An increase (reduction) of the energy scale, keeping the energy thresholds $E_{0}$ fixed, would change the upper limits by $+14 \%(-54 \%)$ above $1 \mathrm{EeV}$ and by $+6 \%(-7 \%)$ above $2,3,5$ and $10 \mathrm{EeV}$. This is a consequence of a different number of photon candidates $\left(_{-4}^{+1}\right.$ in the first bin, unchanged in the others) and of the change in the exposure $\left(\begin{array}{c}-6 \% \\ +7 \%\end{array}\right)$.

\section{CONCLUSIONS AND OUTLOOK}

After few years of operations, the Pierre Auger Observatory has already demonstrated its unique potential for the detection of ultra-high energy photons. Detection has not yet been claimed so far but stringent upper limits have been derived in a wide energy range and using complementary experimental techniques. The presented limits on photon flux in UHECR favor astrophysical scenarios for the origin of the highest energy particles putting severe constraints to the alternative non-acceleration models. These bounds also help reducing the systematic uncertainties on primary mass composition, energy spectrum and proton-air cross-section measurements in the EeV range. The current sensitivity of the Pierre Auger Observatory is at the level of the most optimistic GZK predictions. Updates of the analyses are on-going, both at low and high energies, with growing exposure and improved analyses. Moreover directional photon searches have been started to spot candidate sources.

\section{References}

[1] G. Gelmini, O. Kalashev, D. Semikoz, J. Exp. Theor. Phys. 106 (2008) 1061

[2] V.S. Berezinsky et al. Phys. Lett. B 28 (1969) 423

[3] K. Greisen, Phys. Rev. Lett. 16 (1966) 748. G.T. Zatsepin, V.A. Kuzmin, JETP Lett. 4 (1966) 78

[4] P. Bhattacharjee, G. Sigl, Phys. Rep. 327 (2000) 109

[5] K. Shinozaki et al., Astrophys. J. 571 (2002) L117

[6] M. Ave et al., Phys. Rev. Lett. 85 (2000) 2244. Phys. Rev. D 65 (2002) 063007

[7] A. Glushkov et al., Phys. Rev. D 82 (2002) 041101

[8] M. Risse et al., Phys. Rev. Lett. 95 (2005) 171102

[9] The Pierre Auger Collaboration, Astropart. Phys. 27 (2007) 155

[10] The Pierre Auger Collaboration, Astropart. Phys. 29 (2008) 243

[11] The Pierre Auger Collaboration, Astropart. Phys. 31 (2009) 399

[12] M. Risse, P. Homola, Mod. Phys. Lett. A 22 (2007) 749

[13] J. Alvarez-Muniz and M. Risse for the Pierre Auger Collaboration, G. I. Rubtsov and B. T. Stokes for the Telescope Array Collaboration, these proceedings

[14] The Pierre Auger Collaboration, Nucl. Instr. Meth. Phys. Res. A 523 (2004) 50

[15] The Pierre Auger Collaboration, Nucl. Instr. Meth. Phys. Res. A 613 (2012) 29

[16] The Pierre Auger Collaboration, Nucl. Instr. Meth. Phys. Res. A 620 (2010) 227

[17] L.D. Landau, I. Ya. Pomeranchuk, Dokl. Akad. Nauk SSSR 92, 535 and 735 (1953). A.B. Migdal, Phys. Rev. 103, 1811 (1956)

[18] J. Bellido for the Pierre Auger Collaboration, 31 ${ }^{\text {st }}$ ICRC, Łódź, Poland (7-15 July 2009) \# 124. arXiv:0906.2319 [astro-ph.HE]

[19] G. Ros et al., Astrop. Phys. 35 (2011) 140

[20] M. Settimo for the Pierre Auger Collaboration, 32 ${ }^{\text {nd }}$ ICRC, Beijing, China (11-18 Aug 2011) \# 393. arXiv:1107.4805 [astro-ph.HE]

[21] The Pierre Auger Collaboration, Astropart. Phys. 33 (2010) 108

[22] The Pierre Auger Collaboration, Astropart. Phys. 35 (2012) 591

[23] D. Heck et al., Report FZKA, 60191998

[24] S. Ostapchenko, Phys. Lett. B, 636 (2006) 40

[25] A. Fassò et al., CERN-2005-10 INFN/TC_05/11, SLAC-R-773 (2005) 


\section{UHECR 2012}

[26] The Pierre Auger Collaboration, Astropart. Phys. 34 (2011) 368

[27] F. Salamida for the Pierre Auger Collaboration 32 ${ }^{\text {nd }}$ ICRC, Beijing, China (11-18 Aug 2011) \# 893. arXiv:1107.4809 [astro-ph.HE]

[28] The Pierre Auger Collaboration, Phys. Rev. Lett. 104 (2010) 091101

[29] R. Pesce for the Pierre Auger Collaboration, 32 ${ }^{\text {nd }}$ ICRC, Beijing, China (11-18 Aug 2011) \# 1160. arXiv:1107.4809 [astro-ph.HE]

[30] G.I. Rubtsov for the Telescope Array Collaboration, 32 ${ }^{\text {nd }}$ ICRC, Beijing, China (11-18 Aug 2011) \# 1266

[31] J. Ellis et al., Phys. Rev. D 74 (2006) 115003 